\title{
Abyssomicin E, a Highly Functionalized Polycyclic Metabolite from Streptomyces sp.
}

Xue-Mei Niu, ${ }^{\dagger}$ Sheng-Hong Li, ${ }^{\ddagger}$ Helmar Görls, ${ }^{\S}$ Dieter Schollmeyer, ${ }^{ף}$ Matthias Hilliger, ${ }^{\dagger,}{ }^{\perp}$ Susanne Grabley ${ }^{\dagger}$, and Isabel Sattler ${ }^{*} \dagger$

${ }^{\dagger}$ Leibniz-Institute for Natural Product Research and Infection Biology - Hans-Knöll-Institute, Beutenbergstraße 11a, D-07745 Jena, Germany.

${ }^{\ddagger}$ Max-Planck-Institute for Chemical Ecology, D-07745 Jena, Germany.

$\S$ Institute for Inorganic and Analytical Chemistry, Friedrich Schiller University Jena, Germany.

"Insitute for Organic Chemistry, Johannes Gutenberg University Mainz, Germany.

E-mail isabel.sattler@hki-jena.de 


\section{Supporting Information}

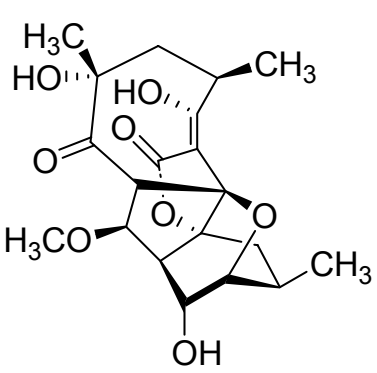

abyssomicin E

(1) Fig. $1^{1} \mathrm{H}$ NMR spectrum of abyssomicin $\mathrm{E}$

(2) Fig. $2{ }^{13} \mathrm{C}$ NMR spectrum of abyssomicin $\mathrm{E}$

(3) Fig. $3{ }^{1} \mathrm{H}-{ }^{1} \mathrm{H}$ COSY spectrum of abyssomicin $\mathrm{E}$

(4) Fig. 4 HMQC spectrum of abyssomicin E

(5) Fig. 5 HMBC spectrum of abyssomicin E

S-7

(6) Fig. 6 NOESY spectrum of abyssomicin E 
Fig. $1^{1} \mathrm{H}$ NMR spectrum of abyssomicin E

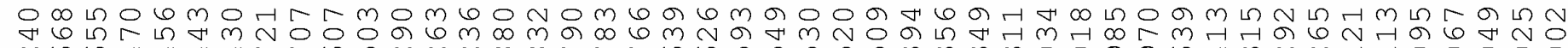

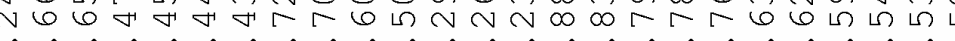

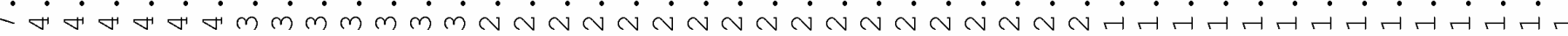
$L L L \perp \perp \perp L \perp L L L L L L L L L L L L L L L L L L L L L L$

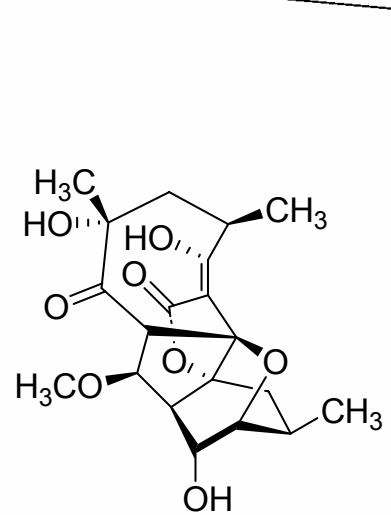

abyssomicin $\mathrm{E}$
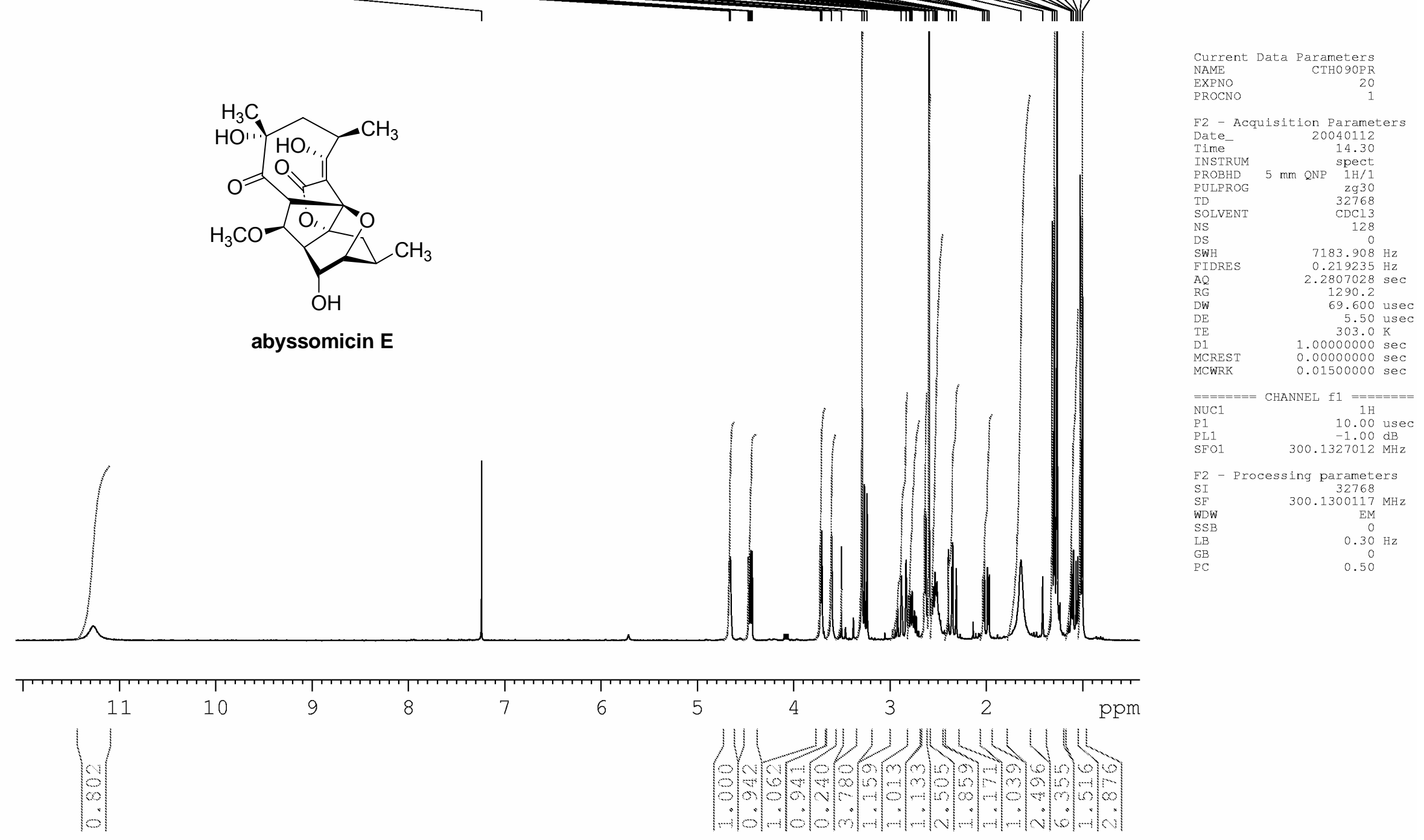
Fig. $2{ }^{13} \mathrm{C}$ NMR spectrum of abyssomicin $\mathrm{E}$

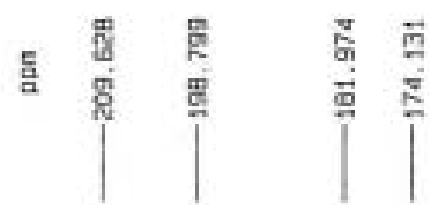

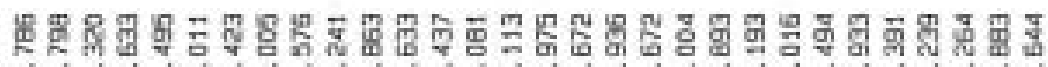

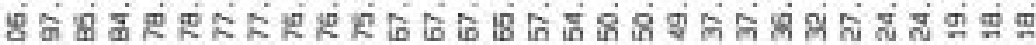
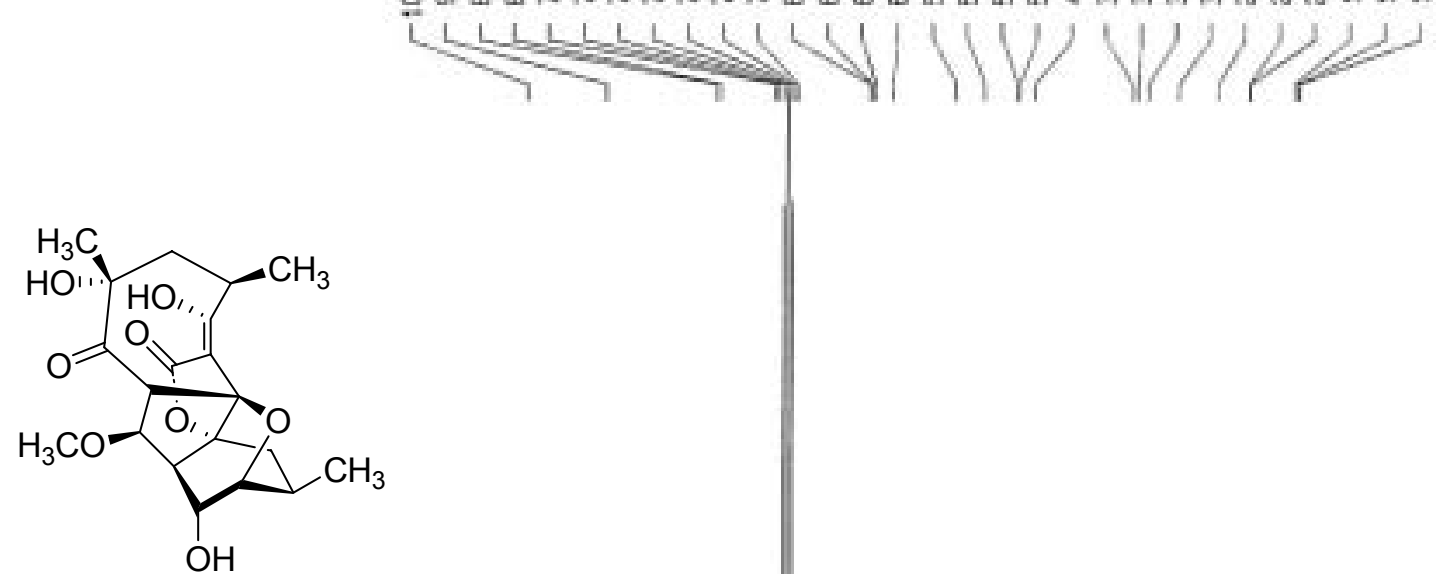

abyssomicin E

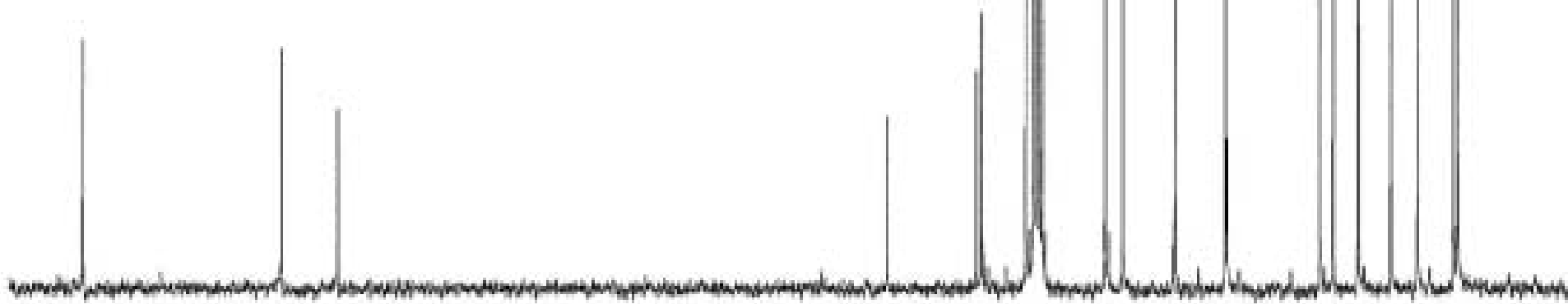

por 200 1 的 1teo 140 120 100 ato ED 10 20

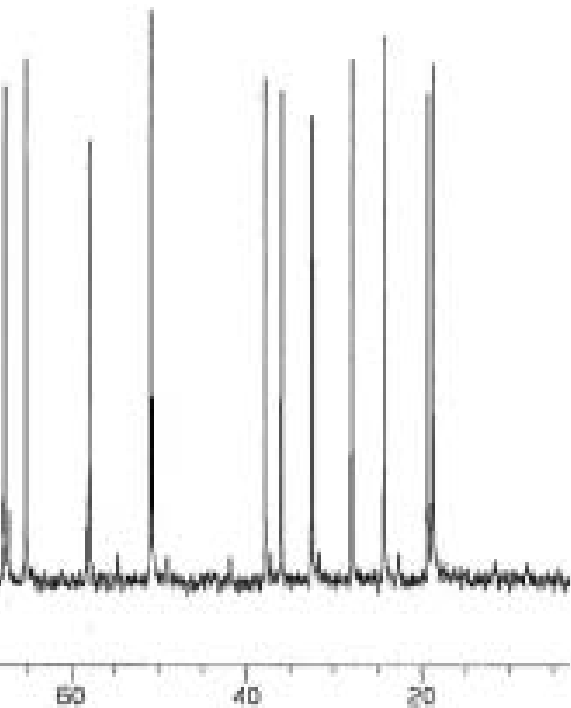

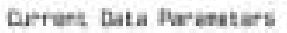

mence

FHSON

hole Bcuetsition Paraseter

Dote- 2tososets

Tine

satso

suraper srect

Eusara

in

MS

SWH $19839.395 \mathrm{~Hz}$

rooes $0.570 \mathrm{mes}$

in D,atesizar sec

In 2 at 400 use

QE 3.05 use

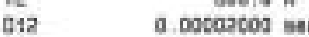

Ris 24,00

a1 0.40tootos sec

orpepse weltzte

PCPOR $\quad$ m7.00 use

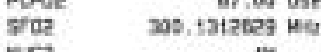

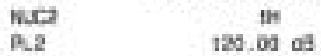

120.02
R.12

$31 \quad 3.00$ use

axi

R.t

ait a. 0900000 se

F2 - Arocessim paraseters

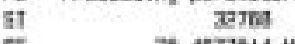

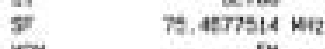

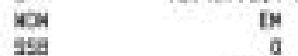

LS $1.00 \mathrm{~Hz}$

to MeA plut taranters

ex 22000

Fip a19 1972 pon

71 10000 as it

rap 3.04: pas

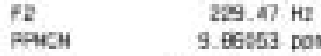

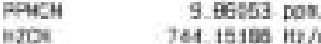


Fig. $3{ }^{1} \mathrm{H}-{ }^{1} \mathrm{H}$ COSY spectrum of abyssomicin E

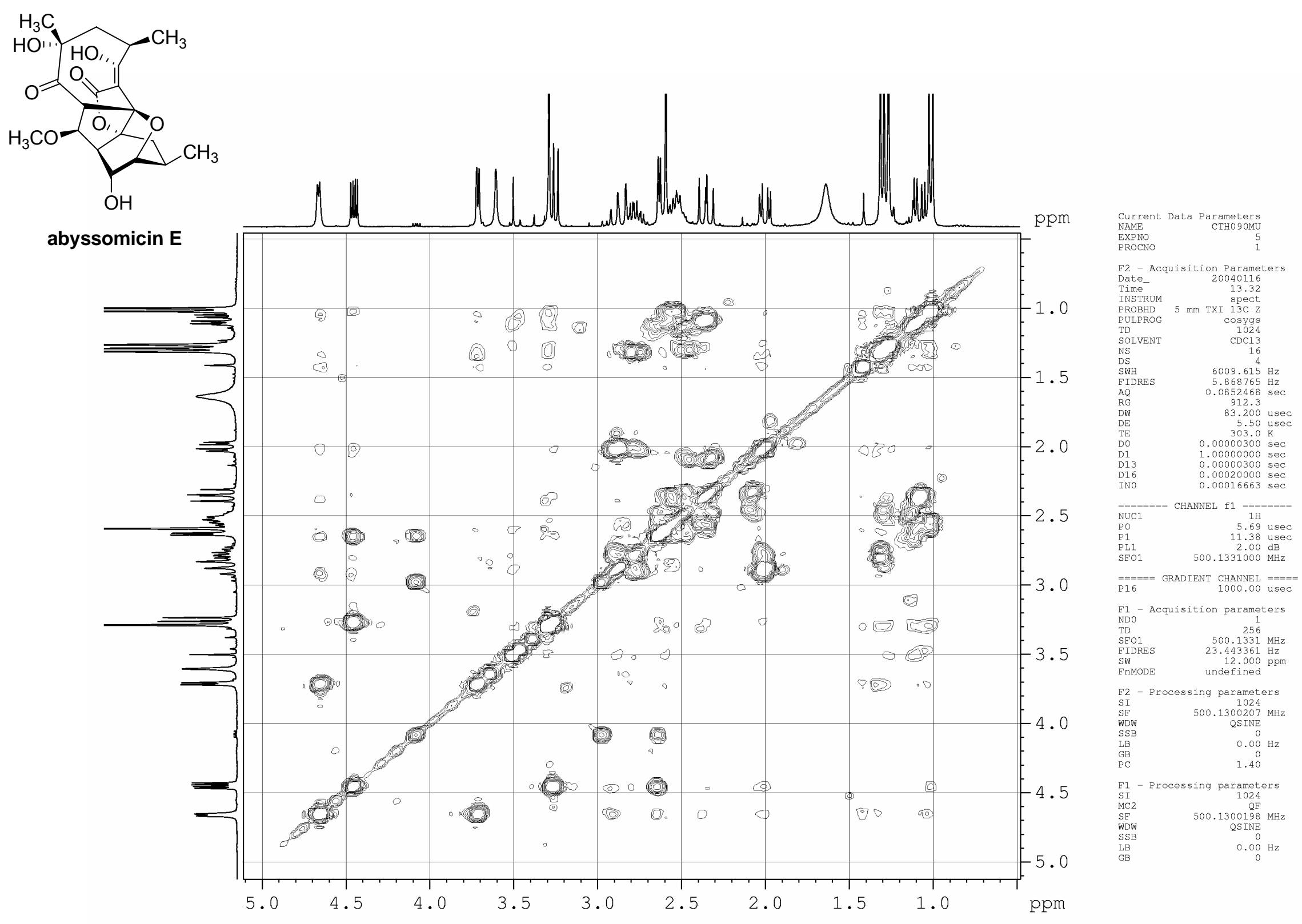


Fig. 4 HMQC spectrum of abyssomicin E
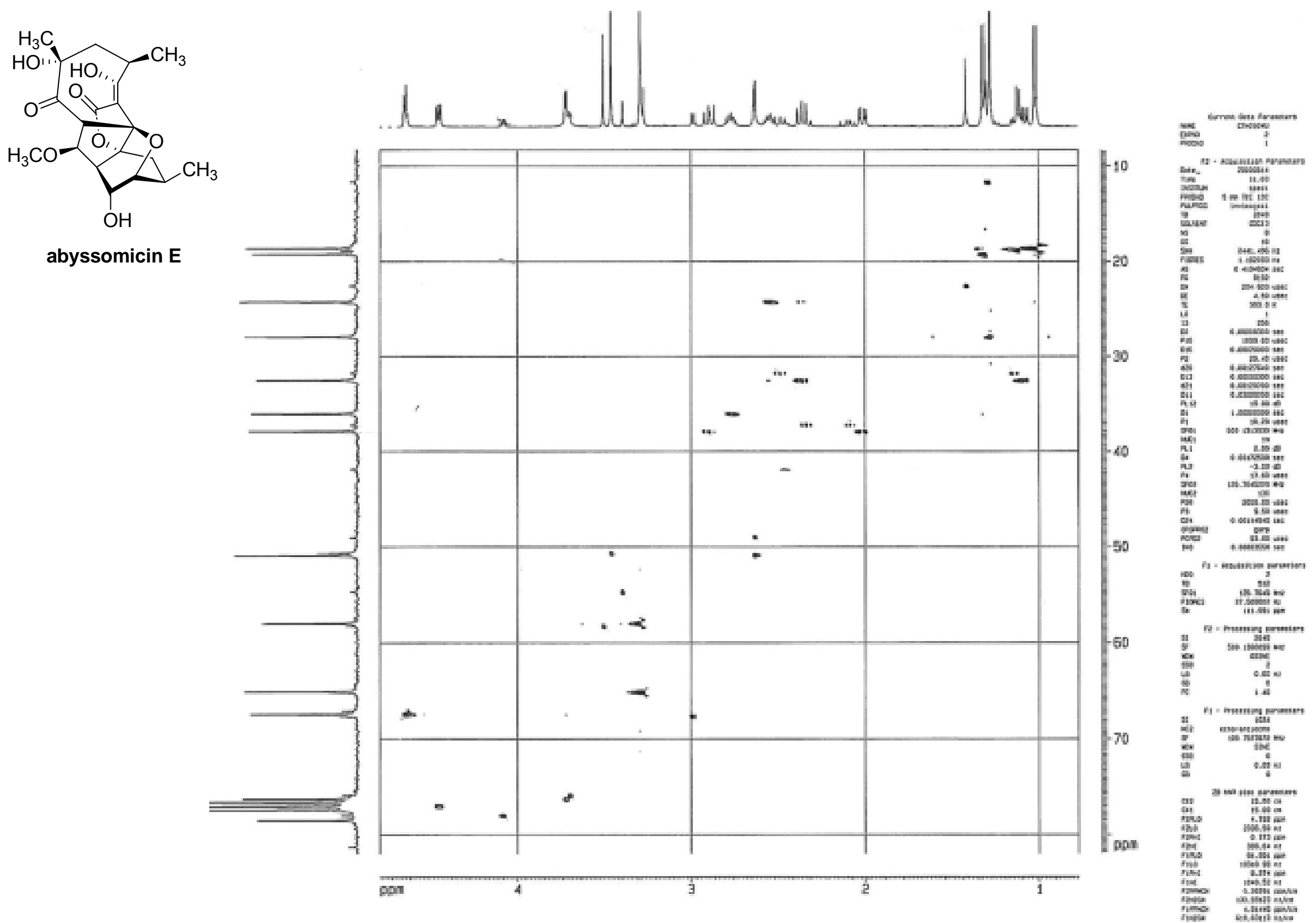
Fig. 5 HMBC spectrum of abyssomicin E

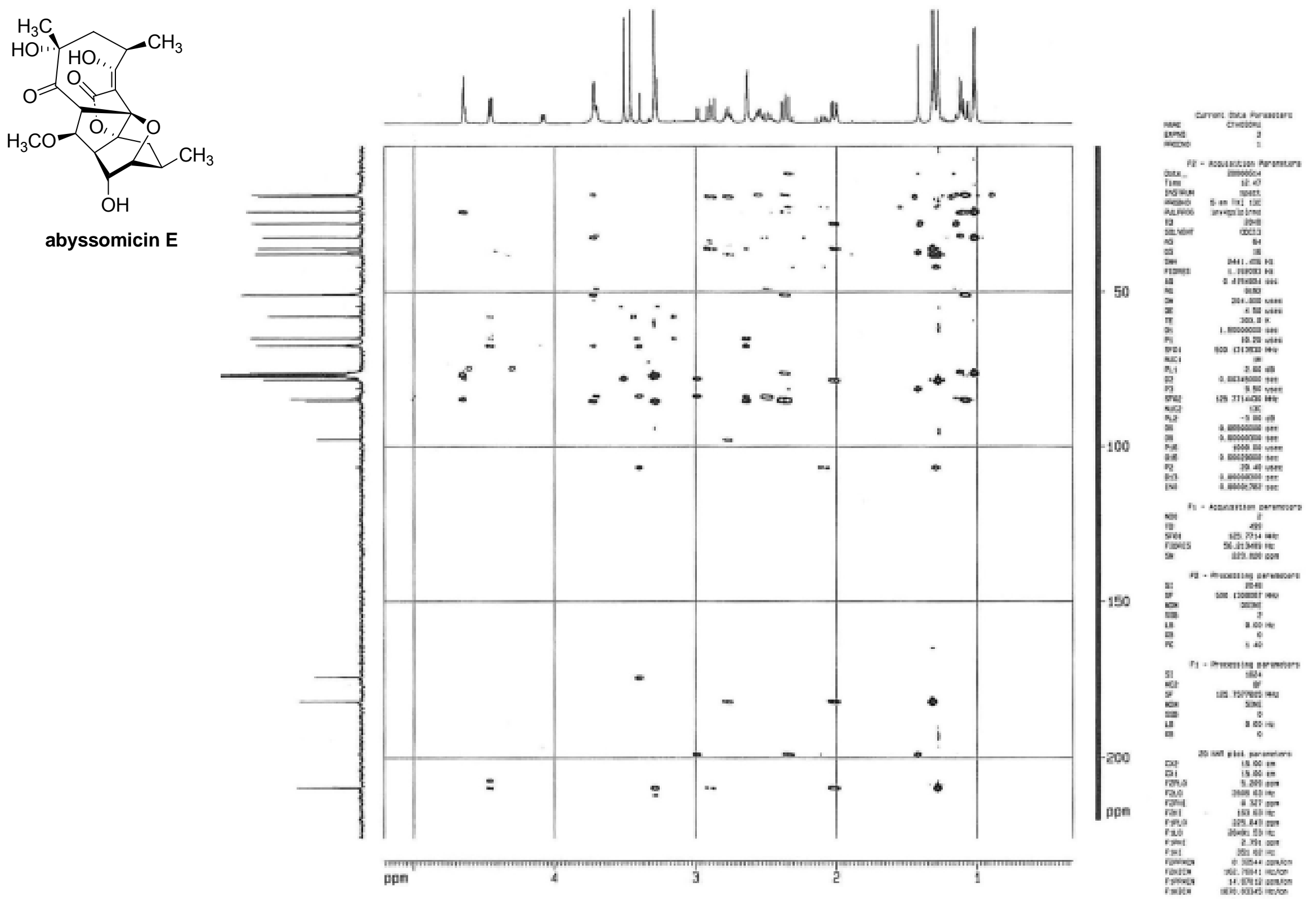


Fig. 6 NOESY spectrum of abyssomicin E

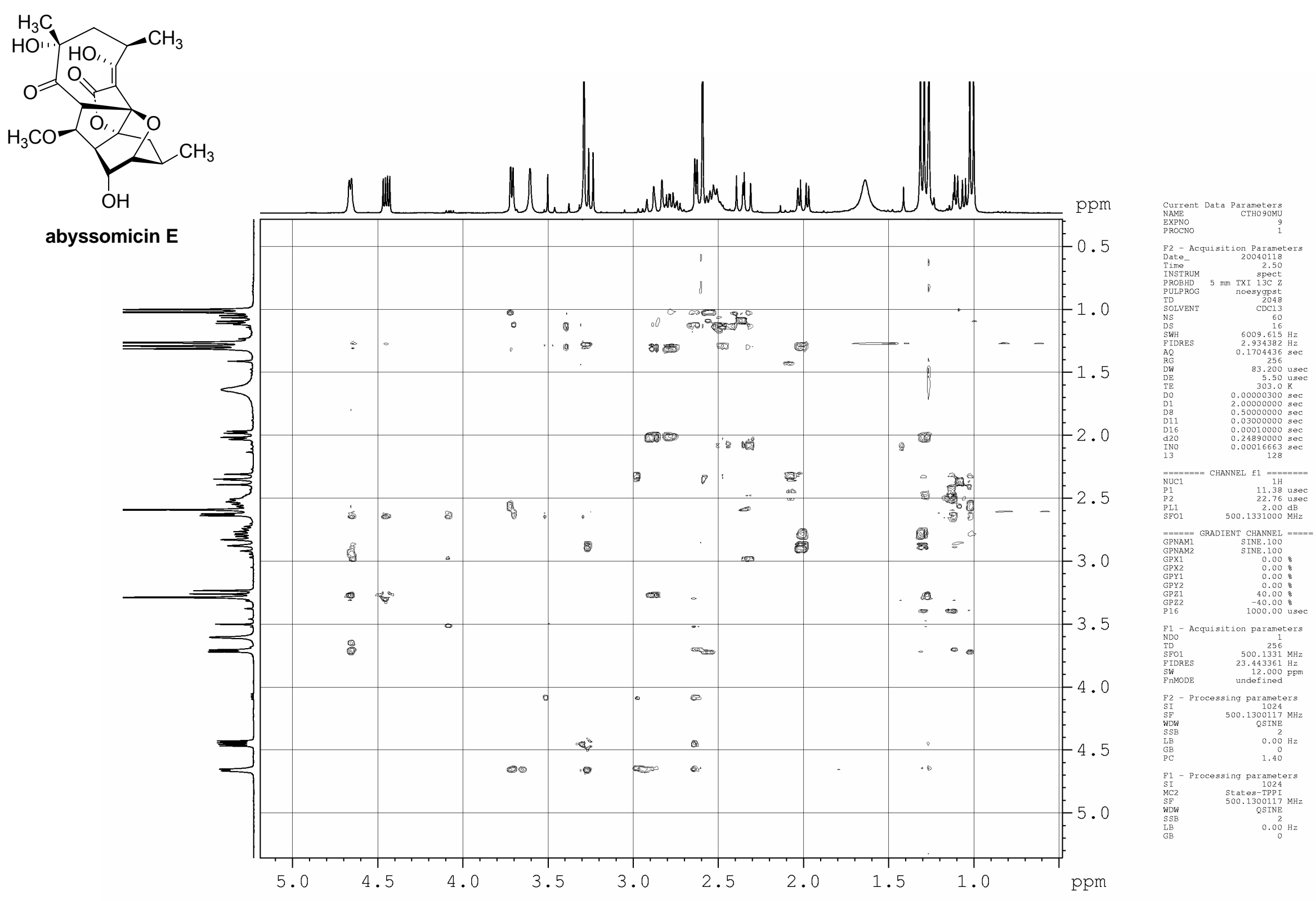

